\title{
Uso de plantas medicinais pela população de Ariquemes, em Rondônia
}

\author{
Maurício Reginaldo A dos Santos; Maria Railda de Lima; Maria das Graças R Ferreira \\ Embrapa Rondônia, C. Postal 406, 78900-970 Porto Velho-RO; mauricio@cpafro.embrapa.br
}

\section{RESUMO}

A utilização de plantas medicinais reflete a realidade de parte da população brasileira, cujo limitado acesso aos programas de saúde pública levou ao desenvolvimento e conservação de um conhecimento etnobotânico rico de informações. Porém, a sabedoria popular carece de sistematização, para que possa ser devidamente utilizada. O objetivo deste trabalho foi relatar o uso de plantas medicinais no município de Ariquemes, em Rondônia, bem como avaliar os padrões sócio-econômicos dos entrevistados em relação à utilização da fitoterapia no seu cotidiano. Para coleta das informações etnobotânicas e etnossociais, foram aplicados questionários estruturados a 44 indivíduos escolhidos por possuírem prestígio junto à comunidade em relação ao conhecimento e uso de plantas medicinais, identificando-se a finalidade, os órgãos e o modo de uso da planta medicinal. Além disso, foi identificada a espécie de cada planta utilizada. Procurou-se ainda correlacionar o conhecimento etnobotânico (inferido a partir do número de citações por indivíduo), com a forma de aquisição dos conhecimentos, aspectos religiosos, educacionais, região de origem, tempo de residência no local e gênero dos entrevistados. Identificaram-se 63 espécies de plantas medicinais em uso pela população, distribuídas em 38 famílias, com maior representatividade para a família Lamiaceae. A parte mais utilizada das plantas foram as folhas e o decocto foi o modo de preparo mais usual. Os estudos etnossociais permitiram inferir que os conhecimentos etnobotânicos da população estudada foram adquiridos principalmente por meio de livros e concentraram-se entre pessoas do gênero masculino, de religiões evangélicas, com nível de escolaridade Fundamental, da $1^{\mathrm{a}}$ à $4^{\mathrm{a}}$ série, provenientes da região Sudeste; e do gênero masculino. O tempo de residência no município não afetou significativamente o conhecimento etnobotânico na população estudada.

Palavras-chave: etnobotânica, fitoterapia, Amazônia.

\author{
ABSTRACT \\ Use of medicinal plants by the population of Ariquemes, in \\ Rondônia State, Brazil
}

The use of medicinal plants shows the reality of part of the Brazilian population whose limited access to the health public programs leads to the development and conservation of a rich ethnobotanic knowledge. However, the popular knowledge needs systematization for adequate utilization. The aim of this work was to study the use of medicinal plants in Ariquemes, Rondônia State, Brazil, and to evaluate the social and economic patterns in relation to the use of phytotherapy. To have access to ethnobotanic and ethnosocial data structured interviews were applied to 44 persons chosen for their prestige in the community in relation to the knowledge and use of medicinal plants, identifying therapeutic purpose, part of the plant used and methods of preparation. Moreover, the species of each used plant was identified. The ethnobotanic knowledge (estimated by the number of citations per informer) was correlated to the knowledge source, religious and educational aspects, region of origin, residence period in Ariquemes and sex of the informers. Sixty-three species of medicinal plants being used by the population were identified, distributed in thirty-eight families, the Lamiaceae being the most representative. The most used part and method of preparation were the leaf and decoction. Ethnosocial studies allowed to infer that the books were the major source of ethnobotanic knowledge; which concentrates in the masculine individuals; and evangelic people; with basic level ( $1^{\text {st }}$ to $4^{\text {th }}$ series $)$; from the Southeast of Brazil. Residence period in Ariquemes did not affect the ethnobotanic knowledge.

Keywords: ethnobotany, phytotherapy, Amazon.

\section{(Recebido para publicação em 9 de outubro de 2006; aceito em 3 de abril de 2008)}

$\mathrm{D}$ urante milênios o homem aprofundou seus conhecimentos empiricamente a fim de melhorar sua alimentação e tratar de suas enfermidades, criando uma inter-relação entre o uso das plantas e sua evolução (Miguel \& Miguel, 2000). Provavelmente a utilização das plantas como medicamento seja tão antiga quanto o próprio homem. Numerosas etapas marcaram a evolução da arte de curar, contudo torna-se difícil delimitá-las com exatidão, já que a medicina esteve por muito tempo associada a práticas mágicas, místicas e ritualísticas (Martins et al., 1995). Atualmente, a Organização Mundial de Saúde considera fundamental que se reali- zem investigações experimentais acerca das plantas utilizadas para fins medicinais e de seus princípios ativos, para garantir sua eficácia e segurança terapêutica (Santos, 2004). Paralelamente, se faz necessário o levantamento das espécies medicinais de cada região fitogeográfica do Brasil, como primeiro passo para a adoção das plantas medicinais nos programas de atenção primária à saúde, o que pode resultar em diminuição de custos e ampliação do número de beneficiados (Matos, 1997).

Tradicionalmente, etnobotânicos de todo o mundo têm registrado plantas, seus usos por populações humanas e formas terapêuticas (no caso de plantas medici- nais). Esse tipo de procedimento proporciona o progresso dos estudos básicos e aplicados, fitoquímicos e farmacológicos, uma vez que fornece a matéria-prima aos pesquisadores de áreas afins e o conjunto de dados necessários para as análises pretendidas. Nesta perspectiva, reconhecer a importância das relações entre o homem e a natureza significa um avanço cognitivo, onde a ciência é utilizada para proteger o patrimônio cultural e a biodiversidade (Albuquerque, 2002).

$\mathrm{Na}$ área da etnobotânica têm sido realizadas pesquisas com comunidades residentes nas regiões de florestas tropicais, com o objetivo de avaliar os recursos vegetais utilizados por estas co- 
munidades e apontar propostas para seu uso sustentado, como forma de preservar e recuperar esses ecossistemas (Silva \& Andrade, 2005). O conhecimento tradicional sobre o uso das plantas é vasto e, em muitos casos, é o único recurso para tratamento da saúde que as populações rurais de países em desenvolvimento têm ao seu alcance. Alguns autores propõem-se a estimar o valor de uso das plantas com a finalidade de apontar as espécies e famílias de preferência da população humana no universo vegetal (Phillips \& Gentry, 1993). No Brasil, considerando a ampla diversidade de espécies vegetais, bem como a riqueza étnico-cultural, o uso popular de plantas medicinais é muito relevante. Por isso, os estudos etnobotânicos são fundamentais, uma vez que possibilitam o resgate e a preservação dos conhecimentos populares das comunidades envolvidas (Garlet \& Irgang, 2001). Conforme ressalta Albuquerque (2002), os informantes devem ser tratados como especialistas, pois são dotados de conhecimentos e fenômenos que nos são desconhecidos e que buscamos compreender.

A utilização de plantas medicinais como alternativa terapêutica vem atingindo um público cada vez maior. Este crescimento requer dos pesquisadores e estudiosos um maior empenho, no sentido de fornecer informações relativas ao sistema produtivo dessas plantas e preparo dos medicamentos, pois nem sempre as normas que garantem a qualidade dos fitoterápicos são cumpridas (Castro \& Ferreira, 2000). A vasta gama de informações sobre o uso de centenas de plantas como remédios, em todos os lugares do mundo, leva à necessidade de se desenvolver métodos que facilitem a enorme tarefa de avaliar cientificamente o valor terapêutico de espécies vegetais (Elisabetsky, 2001).

O objetivo deste trabalho foi relatar o uso de plantas medicinais no município de Ariquemes, em Rondônia, contribuindo assim para auxiliar no resgate do conhecimento tradicional. Procurouse também estabelecer uma correlação entre os padrões sócio-econômicos dos entrevistados e a utilização da fitoterapia como prática em seu cotidiano.

\section{MATERIAL E MÉTODOS}

A pesquisa de campo foi realizada no município de Ariquemes, em
Rondônia, localizado, de acordo com o Zoneamento Sócio-Econômico-Ecológico do Estado de Rondônia (ZSEE), na zona 01 (áreas de usos agropecuários, agroflorestais e florestais) (Millikan, 1998). Conta atualmente com aproximadamente 84 mil habitantes (projeção IBGE, 2005) e com uma área de $4.706,70 \mathrm{~km}^{2}$, localizando-se $198 \mathrm{~km}$ ao Sul da capital Porto Velho. A economia do município está voltada para o setor primário: agricultura, pecuária, extrativismo vegetal e mineral.

Para o levantamento dos dados foram realizadas entrevistas de abril a dezembro de 2005. O entrevistador empregou diálogos para direcionar a conversa, baseando-se em questionários já estruturados. Buscou-se dar mais ênfase aos dados etnobotânicos, como indicação terapêutica, parte da planta utilizada nas preparações e modo de preparo. Os 44 informantes foram escolhidos por possuírem prestígio junto à comunidade em relação ao conhecimento e uso de plantas medicinais e foram entrevistados individualmente, em suas residências, nos dias laborais e finais de semana.

Foram obtidas médias, dividindo-se o número de citações (somatório do número de todas as citações de utilizações de plantas com alguma finalidade terapêutica mencionadas pelos entrevistados) pelo número de entrevistados, em relação aos fatores: forma de aquisição dos conhecimentos (pessoas conhecidas, livros, parentes e pastorais), religião (adventista, católico, evangélico, outros e sem religião), região de origem (Centro-Oeste, Nordeste, Norte, Sudeste e Sul), nível de escolaridade (analfabetos, alfabetizados, Ensino Fundamental da $1^{\mathrm{a}}$ à $4^{\mathrm{a}}$ série, Ensino Fundamental da $5^{\mathrm{a}}$ à $8^{\mathrm{a}}$ série, Ensino Médio e Ensino Superior), tempo de residência no local (até 3 anos; de 4 a 6 anos; de 7 a 9 anos; de 10 a 12 anos; de 13 a 15 anos; de 16 a 18 anos; de 19 a 21 anos; de 22 a 24 anos; de 25 a 27 anos; e de 28 a 30 anos), e gênero dos entrevistados. A utilização deste procedimento teve por objetivo a identificação de como está distribuído o conhecimento sobre fitoterápicos nesta população, em relação aos fatores mencionados. Por exemplo, dentro do fator "nível de escolaridade", para se inferir sobre o conhecimento etnobotânico dentro do grupo "analfabetos" da população, dividiu-se o número de citações (utilizações de plantas com finalidade terapêutica mencionadas em entrevistas com os analfabetos da população) pelo número de indivíduos analfabetos, obtendo-se a média de citações por indivíduo. Da mesma forma, foram obtidas médias para os outros grupos populacionais, e estas foram comparadas entre si, dentro de cada fator, utilizando-se o teste $\mathrm{t}$ de Student $(p<0,05)$.

Não foi possível a identificação taxonômica de todos os espécimes, devido à indisponibilidade de material vegetal adequado para a classificação à época do levantamento. As plantas que apresentavam floração e frutificação foram coletadas, posteriormente herborizadas, seguindo o procedimento de prensagem entre jornais, papelão e corrugado, em prensa de madeira, sendo que cada espécime foi identificada com número de coleta, data, local e nome do coletor. Após esse processo, o material foi colocado em estufa elétrica para desidratação, por um período de três dias. Depois de desidratado, o material vegetal foi descrito e identificado com auxílio de lupa, literatura especializada, ou por comparação com material já identificado e, posteriormente, incorporado ao acervo do herbário Dr. Ary Tupinambá Penna Pinheiro, pertencente à Faculdade São Lucas (FSL), município de Porto Velho, Rondônia.

\section{RESULTADOS E DISCUSSÃO}

Foram coletados 77 espécimes, sendo identificadas 63 espécies distribuídas em 38 famílias botânicas (Tabela 1). As famílias mais representativas em número de espécies foram Lamiaceae, com sete espécies, e Asteraceae, Leguminosae e Compositae, com quatro espécies cada, enquanto as demais tiveram apenas uma ou duas espécies mencionadas. Foram constatadas oito formas de preparo dos fitoterápicos, sendo a mais utilizada o decocto, ou seja, o cozimento da parte vegetal em água e, em segundo lugar, o infuso, que consiste na submersão da parte vegetal em água, logo após a fervura desta. Isto tam- 
bém foi observado por Rodrigues (1998) durante levantamento florístico e etnobotânico de plantas medicinais do Cerrado, na Região do Alto Rio Grande, em Minas Gerais. Destacou-se também nesse levantamento o uso combinado de plantas com outros ingredientes como: poejo (Mentha pulegium L.) com mel, babosa (Aloe vera (L.) Burn. F.) com mel e cachaça, pé-de-galinha (Eleusine indica (L.) Gaertn) com pedaço de cupim e álcool. Outro fato constatado foi o uso de várias ervas medicinais com chimarrão, conforme já anteriormente observado por Garlet \& Irgang (2001), em Cruz Alta, no Rio Grande do Sul.

As plantas mencionadas com maior freqüência foram crajiru (Arrabidaea chica (Bonpl.) B. Verl.), boldo (Plectranthus barbatus Andrews), hortelã (Mentha sp.), erva-cidreira (Lippia alba (Mill.) N. E. Br), erva-de-SantaMaria (Chenopodium ambrosioides L.), poejo (Mentha pulegium L.), hortelãgrande (Plectranthus amboinicus (Lour) Spreng.), algodão (Gossypium hirsitum L.), babosa (Aloe vera (L.) Burn. F.) e alfavaca (Ocimum selloi Benth.). Também foram mencionadas com freqüência a associação de mais de uma planta nas formulações, dentre elas romã (Punica granatum L.) e gengibre (Zingiber officinale Roscoe), chapéude-couro (Echinodorus grandiflorus Mitch) e crajiru (Arrabidaea chica (Bonpl.) B. Verl.), cana-do-brejo (Costus spicatus (Jack) SW) e pata-devaca (Bauhinia forficata Link) e abacate (Persea americana Mill) e quebra-pedra (Phyllanthus niruri L.). Observações semelhantes foram constatadas por Ming \& Amaral Júnior (2005) em estudo realizado na reserva extrativista "Chico Mendes".

Observou-se que não há uma padronização com referência à quantidade de planta a ser empregada nas preparações, sendo indicado um punhadinho, uma mão cheia, um maço, entre outras. Quanto à parte vegetal utilizada nas preparações, observou-se uma maior utilização das folhas. É interessante observar que as folhas, além de geralmente concentrarem grande parte dos princípios ativos da plantas, podem ser coletadas sem causar grandes danos às plantas, garantindo sua preservação. Toda informação colhida foi transcrita literalmente, procedimento também adotado por Radomski \& Wisniewski (2004), mantendo-se expressões como tiriça (icterícia), afinar o sangue (reduzir as concentrações de ácidos graxos no sangue), pano branco (doença de pele causada pelo fungo Pityrosporum ovale) e chiado no peito (asma), termos utilizados pelos entrevistados (Tabela 1).

Foram registradas 237 citações, nas quais foi relatada a utilização de várias partes vegetais das 63 espécies, em oito formas de preparo, com diferentes finalidades terapêuticas.

Com relação às diferentes religiões praticadas por cada comunidade, podese inferir uma maior concentração de conhecimentos nos evangélicos e católicos, com médias de 7,4(a) e 6,7(a) citações por entrevistado, respectivamente, seguidos pelos entrevistados sem religião, com 5,6(b), e os adventistas, com 4,3(c). Esses dados nos permitem constatar que há uma interação entre os conhecimentos etnobotânicos e as religiões. É importante ressaltar que grande parte dos entrevistados que se declararam evangélicos, eram ex-católicos, convertidos recentemente. De acordo com Camargo (1998), as pesquisas na área da medicina popular em todos os segmentos da sociedade demonstram uma constante vinculação com crenças religiosas.

Com relação à origem dos entrevistados, constatou-se que as pessoas advindas do Sudeste do país detinham mais informações em relação às demais regiões, com média de 8,5(a) citações por entrevistado; em seguida, a região Nordeste apresentou média de 7,3(b); a região Centro-Oeste $7,0(\mathrm{~b})$; a região Norte 5,6(c); e a região Sul 4,7(c). Notase que, embora a região Norte abrigue uma enorme biodiversidade, inclusive no que diz respeito a plantas medicinais, os conhecimentos etnobotânicos não se concentraram nos indivíduos originários do Norte. Isto se deve provavelmente ao fato de que a maioria das plantas estudadas foram trazidas de outras regiões. Das 63 espécies mencionadas, apenas sete são amazônicas: crajiru (Arrabidea chica (Bonpl.) B. Verl.), cana-do-brejo (Costus spicatus
(Jack)SW), terramicina (Althernanthera dentata (Moench) Stuchlik), copaíba (Copaifera langsdorfii Desf.), coqueirinho (Elleutherine bulbosa Mill.), cupuaçu (Theobroma grandiflora Willd.) e insulina (Cissus verticilata L.), o que sugere que a cultura etnobotânica sobre as plantas amazônicas está se perdendo, sendo um dos motivos a carência de estudos na região, tanto em relação ao resgate cultural quanto à descoberta de novas plantas como prováveis fontes de substâncias bioativas de interesse, devido à falta de incentivo por parte das instituições acadêmicas e de pesquisa. Em um recente levantamento realizado por Fernandes (2004) acerca de trabalhos apresentados nos simpósios de plantas medicinais, de 1972 a 1998, as regiões mais participativas, em termos de número de trabalhos apresentados, foram Sudeste, Sul e Nordeste, sendo que as regiões Centro-Oeste e Norte apresentaram uma parcela pouco significativa.

Em relação ao nível de escolaridade, o conhecimento e uso de plantas medicinais predominou no nível de Ensino Fundamental da $1^{\mathrm{a}}$ à $4^{\mathrm{a}}$ série, no nível de Ensino Fundamental da $5^{\mathrm{a}}$ à $8^{\mathrm{a}}$ série e no Ensino Médio, com médias de 7,3(a), 6,8(a) e 6,7(a) citações por entrevistado, respectivamente. Entre os analfabetos, obteve-se 5,6(b) e no nível de Ensino Superior foi observada a menor média, 3,0(c) citações por entrevistado. Assim, observa-se que o conhecimento sobre plantas medicinais apresenta uma tendência a diminuir com o nível de escolaridade. Está claro que o nível de escolaridade está associado a condições econômicas. Assim, a relação entre o baixo nível de escolaridade e a maior familiarização com o poder medicinal de espécies vegetais pode refletir a busca, devido ao baixo poder aquisitivo, de formas alternativas de tratar as doenças, que não envolvam a compra de medicamentos caros. Talvez seja possível inferir também que o nível crescente de escolaridade envolve uma certa massificação dos costumes, principalmente frente à globalização, o que levaria a uma perda gradual dos hábitos ancestrais relacionados à fitoterapia.

Os dados relacionados à aquisição dos conhecimentos sobre o uso das plan- 
Tabela 1. Nomes vulgares e científicos, famílias botânicas, indicações, partes utilizadas e modos de preparo de plantas medicinais utilizadas pela população de Ariquemes, em Rondônia (vulgar and scientific names, botanic families, indications, used parts of the plants and methods of preparation of used medicinal plants for the population of Ariquemes, in Rondônia). Porto Velho, Embrapa Rondônia, 2005.

\begin{tabular}{|c|c|c|c|c|}
\hline Nome vulgar - Científico & Família & Indicação $^{1}$ & Parte utilizada $^{1}$ & Modo de preparo ${ }^{1}$ \\
\hline $\begin{array}{l}\text { Abacate - Persea } \\
\text { americana Mill }\end{array}$ & Lauraceae & a- rins & $\begin{array}{l}\text { a- folhas } \\
\text { b- semente }\end{array}$ & $\begin{array}{l}\text { a- infuso } \\
\text { b- decocto }\end{array}$ \\
\hline Acerola - Malphigia glabra L. & Malpighiaceae & a- gripe & a- folhas & a- decocto e melado \\
\hline $\begin{array}{l}\text { Alecrim - Rosmarinus } \\
\text { officinalis L. }\end{array}$ & Lamiaceae & $\begin{array}{l}\text { a- coração, febre, } \\
\text { calmante, menstruação } \\
\text { irregular }\end{array}$ & a- folhas & a- infuso \\
\hline $\begin{array}{l}\text { Alfavaca - Ocimum selloi } \\
\text { Benth. }\end{array}$ & Lamiaceae & a- gripe & a- folhas & $\begin{array}{l}\text { a- decocto e infuso, } \\
\text { decocto para banho }\end{array}$ \\
\hline $\begin{array}{l}\text { Algodão - Gossypium } \\
\text { hirsutum L. }\end{array}$ & Malvaceae & $\begin{array}{l}\text { a- antinflamatório, } \\
\text { infecção } \\
\text { b- baque } \\
\text { c- hepatite }\end{array}$ & $\begin{array}{l}\text { a- folhas } \\
\text { b- folhas } \\
\text { c- raízes }\end{array}$ & $\begin{array}{l}\text { a- sumo, decocto } \\
\text { b- cataplasma } \\
\text { c- decocto }\end{array}$ \\
\hline Alho - Allium sativum L. & Liliaceae & $\begin{array}{l}\text { a- gripe, febre, pressão } \\
\text { alta }\end{array}$ & a- bulbo & a- decocto e infuso \\
\hline $\begin{array}{l}\text { Babosa - Aloe vera (L.) } \\
\text { Burn. F. }\end{array}$ & Liliaceae & $\begin{array}{l}\text { a- queimadura, } \\
\text { cicatrizante, cabelo } \\
\text { b- câncer, úlcera }\end{array}$ & $\begin{array}{l}\text { a- folhas } \\
\text { b- folhas }\end{array}$ & $\begin{array}{l}\text { a- passar no local } \\
\text { b- batido com mel }\end{array}$ \\
\hline $\begin{array}{l}\text { Boldo - Plectranthus } \\
\text { barbatus Andrews }\end{array}$ & Lamiaceae & a- estomago, fígado & a- folhas & a- sumo e infuso \\
\hline $\begin{array}{l}\text { Cajueiro - Anacardium } \\
\text { occidentale L. }\end{array}$ & Anacardiaceae & $\begin{array}{l}\text { a- diarréia, infecção } \\
\text { b- cicatrizante }\end{array}$ & $\begin{array}{l}\text { a- folhas } \\
\text { b- entrecasca }\end{array}$ & $\begin{array}{l}\text { a- decocto e infuso } \\
\text { b- decocto }\end{array}$ \\
\hline $\begin{array}{l}\text { Cana-do-brejo - Costus } \\
\text { spicatus (Jack) SW }\end{array}$ & Zingiberaceae & a- rins & a- folhas, hastes & a- decocto e infuso \\
\hline $\begin{array}{l}\text { Cancerosa - Maytenus } \\
\text { ilicifolia Reissek }\end{array}$ & Celastraceae & $\begin{array}{l}\text { a- câncer, úlcera } \\
\text { b- câncer de pele } \\
\text { c- gripe }\end{array}$ & $\begin{array}{l}\text { a- folhas } \\
\text { b- rizoma } \\
\text { c- rizoma }\end{array}$ & $\begin{array}{l}\text { a- decocto } \\
\text { b- banho local } \\
\text { c- xarope }\end{array}$ \\
\hline $\begin{array}{l}\text { Canela - Cinnamomum } \\
\text { zeylanicum Breyn. }\end{array}$ & Lauraceae & a- vômito, calmante & $\begin{array}{l}\text { a- folhas } \\
\text { b- entrecasca }\end{array}$ & $\begin{array}{l}\text { a- infuso } \\
\text { b- decocto }\end{array}$ \\
\hline $\begin{array}{l}\text { Capim cidreira - } \\
\text { Cimbopogon citratus (DC) } \\
\text { Stapf. }\end{array}$ & Gramineae & $\begin{array}{l}\text { a- calmante, pressão alta, } \\
\text { gripe }\end{array}$ & a- folhas & a- decocto e infuso \\
\hline $\begin{array}{l}\text { Carambola - Averrhoa } \\
\text { carambola L. }\end{array}$ & Oxalidaceae & $\begin{array}{l}\text { a- rins, colesterol, } \\
\text { emagrecedor } \\
\text { b- colesterol }\end{array}$ & $\begin{array}{l}\text { a- folhas } \\
\text { b- fruto }\end{array}$ & $\begin{array}{l}\text { a- decocto } \\
\text { b- suco }\end{array}$ \\
\hline $\begin{array}{l}\text { Carqueja - Baccharis } \\
\text { trimera (Less.) DC }\end{array}$ & Compositae & $\begin{array}{l}\text { a- estômago, menopausa, } \\
\text { emagrecedor }\end{array}$ & a- folhas & a- decocto e infuso \\
\hline $\begin{array}{l}\text { Carqueja doce - } \\
\text { Baccharis articulata }\end{array}$ & Compositae & $\begin{array}{l}\text { a- estômago, } \\
\text { emagrecedor }\end{array}$ & a- folhas & a- infuso \\
\hline $\begin{array}{l}\text { Chapéu-de-couro - } \\
\text { Echinodorus grandiflorus } \\
\text { Mitch }\end{array}$ & Alismataceae & $\begin{array}{l}\text { a- reumatismo, pressão } \\
\text { alta, infecção }\end{array}$ & a- folhas & a- decocto e infuso \\
\hline $\begin{array}{l}\text { Chicória - Cichorium } \\
\text { intibus L. }\end{array}$ & Compositae & $\begin{array}{l}\text { a- pressão alta, cólica } \\
\text { menstrual }\end{array}$ & a- folhas & a- infuso \\
\hline Coco - Cocus nucifera L. & Arecaceae & a- anemia, gastrite, úlcera & a- bucha & a- decocto e infuso \\
\hline $\begin{array}{l}\text { Confrei - Symphytum } \\
\text { officinale }\end{array}$ & Borraginaceae & $\begin{array}{l}\text { a- infecção, rins, gastrite } \\
\text { b- ferimento }\end{array}$ & $a$ - folhas $b$ - folhas & $\begin{array}{l}\text { a- decocto e infuso } \\
\text { b- aplicar no local }\end{array}$ \\
\hline $\begin{array}{l}\text { Copaíba - Copaifera } \\
\text { langsdorfii Desf. }\end{array}$ & Leguminosae & $\begin{array}{l}\text { a- infecção urinária } \\
\text { b- chiado no peito }{ }^{2}\end{array}$ & $\begin{array}{l}\text { a- entrecasca } \\
\text { b- óleo }\end{array}$ & $\begin{array}{l}\text { a- decocto } \\
\text { b- óleo }\end{array}$ \\
\hline $\begin{array}{l}\text { Coqueirinho - Eleutherine } \\
\text { bulbosa (Mill.)Urb. }\end{array}$ & Iridaceae & $\begin{array}{l}\text { a- diarréia sanguinolenta }{ }^{3} \text {, } \\
\text { dentição, hemorróida }\end{array}$ & a- bulbo & a- infuso \\
\hline $\begin{array}{l}\text { Cordão de frade - } \\
\text { Leonotis nepetaefolia }(L .) \\
\text { R. BR. }\end{array}$ & Lamiaceae & a- rins, estômago & a- folhas e flores & a- decocto \\
\hline $\begin{array}{l}\text { Crajiru - Arrabidacea chica } \\
\text { (Bonpl.) B. Verl. }\end{array}$ & Bignoniaceae & $\begin{array}{l}\text { a- inflamação, rins, } \\
\text { infecção }\end{array}$ & a- folhas & a- decocto e infuso \\
\hline
\end{tabular}

${ }^{1}$ As letras de referência indicam conexão entre os itens Indicação, Parte Utilizada e Modo de Preparo. (The reference letters indicate connection between itens Indication, Used Part of the Plant and Method of Preparation); ${ }^{2}$ Asma (asthma); ${ }^{3}$ Melena (melena); ${ }^{4}$ Icterícia (jaundice); ${ }^{5}$ Reduzir as concentrações de ácidos graxos no sangue (to reduce the fatty acids concentrations of the blood); ${ }^{6}$ Doença de pele causada pelo fungo Pityrosporum ovale (skin disease caused for the fungus Pityrosporum ovale). 
Tabela 1. (Continuação)

\begin{tabular}{|c|c|c|c|c|}
\hline Nome vulgar - Científico & Família & Indicação $^{1}$ & Parte utilizada $^{1}$ & Modo de preparo ${ }^{1}$ \\
\hline $\begin{array}{l}\text { Cupuaçu - Theobroma } \\
\text { grandiflorum Willd. }\end{array}$ & Sterculiaceae & a- pressão alta & a- folhas & a- infuso \\
\hline $\begin{array}{l}\text { Erva cidreira - Lippia alba } \\
\text { (Mill.) N. E. Br }\end{array}$ & Verbenaceae & a- calmante, gripe, febre & a- folhas & a- decocto ou infuso \\
\hline $\begin{array}{l}\text { Erva de bicho - Solanum } \\
\text { americanum (Mill.) }\end{array}$ & Solanaceae & a- mioma & a- folhas & a- decocto \\
\hline $\begin{array}{l}\text { Erva de Santa Maria - } \\
\text { Chenopodium } \\
\text { ambrosioides L. }\end{array}$ & Chenopodiaceae & $\begin{array}{l}\text { a- verme, tuberculose } \\
\text { b- baque }\end{array}$ & $\begin{array}{l}\text { a- folhas, sementes } \\
\text { b- folhas }\end{array}$ & $\begin{array}{l}\text { a- batido c/ leite } \\
\text { b- cataplasma }\end{array}$ \\
\hline $\begin{array}{l}\text { Erva-doce - Fueniculum } \\
\text { vulgare Mill }\end{array}$ & Apiaceae & a- prisão de ventre & a- sementes & a- infuso e decocto \\
\hline $\begin{array}{l}\text { Figatil - Vernonia } \\
\text { condensata Baker }\end{array}$ & Asteraceae & a- fígado & a- folhas & a- infuso e macerado \\
\hline $\begin{array}{l}\text { Gengibre - Zingiber } \\
\text { officinale Roscoe }\end{array}$ & Zingiberaceae & $\begin{array}{l}\text { a- garganta, asma } \\
\text { b- gripe }\end{array}$ & $\begin{array}{l}\text { a- rizoma } \\
\text { b- rizoma }\end{array}$ & $\begin{array}{l}\text { a- decocto e infuso } \\
\text { b- xarope }\end{array}$ \\
\hline Goiaba - Psidium guajava L. & Myrtaceae & $\begin{array}{l}\text { a- diarréia, dor de barriga } \\
\text { b- diarréia }\end{array}$ & $\begin{array}{l}\text { a- folhas, brotos } \\
\text { b- entrecasca }\end{array}$ & $a$ - infuso b- decocto \\
\hline $\begin{array}{l}\text { Guaco - Mikania glomerata } \\
\text { Spreng. }\end{array}$ & Compositae & a- gripe & a- folhas & a- infuso e melado \\
\hline $\begin{array}{l}\text { Guiné - Petiveria } \\
\text { alliaceae L. }\end{array}$ & Phytolaccaceae & $\begin{array}{l}\text { a- reumatismo, dor no } \\
\text { corpo } \\
\text { b- malária, dor de cabeça }\end{array}$ & $\begin{array}{l}\text { a- folhas } \\
\text { b- folhas }\end{array}$ & $\begin{array}{l}\text { a- macerado com álcool } \\
\text { b- decocto }\end{array}$ \\
\hline Hortelã - Mentha sp. & Lamiaceae & $\begin{array}{l}\text { a- gripe } \\
\text { b- verme }\end{array}$ & $\begin{array}{l}\text { a- folhas } \\
\text { b- folhas }\end{array}$ & $\begin{array}{l}\text { a- decocto e melado } \\
\text { b- infuso }\end{array}$ \\
\hline $\begin{array}{l}\text { Hortelã-grande - } \\
\text { Plectranthus amboinicus } \\
\text { (Lour) Spreng. }\end{array}$ & Crassulaceae & $\begin{array}{l}\text { a- gripe } \\
\text { b- machucado, ferimento } \\
\text { c- verme }\end{array}$ & $\begin{array}{l}\text { a- folhas } \\
\text { b- folhas } \\
\text { c- folhas }\end{array}$ & $\begin{array}{l}\text { a- melado } \\
\text { b- sumo } \\
\text { c- decocto }\end{array}$ \\
\hline Inhame - Dioscorea sp & Dioscoreaceae & a- reforço alimentar & a- batata & a- cozido \\
\hline $\begin{array}{l}\text { Insulina - Cissus } \\
\text { verticilata L. }\end{array}$ & Vitaceae & a- diabete & a- folhas & a- infuso \\
\hline $\begin{array}{l}\text { Jambolão - Syzygium } \\
\text { cumini ( L.) Skeel }\end{array}$ & Myrtaceae & a- infecção & a- folhas & a- decocto e sumo \\
\hline $\begin{array}{l}\text { Jatobá - Hymenaea } \\
\text { courbaril L. }\end{array}$ & Leguminosae & $\begin{array}{l}\text { a- gripe, anemia, } \\
\text { inflamação, malária }\end{array}$ & a- entrecasca & a- decocto \\
\hline $\begin{array}{l}\text { Jucá - Caelsapinia ferrea } \\
\text { Mart. }\end{array}$ & Leguminosae & $\begin{array}{l}\text { a- inflamação, malária } \\
\text { b- anemia }\end{array}$ & $\begin{array}{l}\text { a- casca do fruto } \\
\text { b- casca do fruto }\end{array}$ & $\begin{array}{l}\text { a- decocto } \\
\text { b- macerado }\end{array}$ \\
\hline Lima - Citrus limetta L. & Rutaceae & a- tiriça ${ }^{4}$ & a- folhas & a- decocto para banho \\
\hline $\begin{array}{l}\text { Limão - Citrus limon (L.) } \\
\text { Burm. F. }\end{array}$ & Rutaceae & $\begin{array}{l}\text { a- gripe } \\
\text { b- afinar o sangue } \\
\text { c- pressão alta }\end{array}$ & $\begin{array}{l}\text { a- folhas } \\
\text { b- fruto } \\
c \text { - fruto }\end{array}$ & $\begin{array}{l}\text { a- infuso } \\
\text { b- suco } \\
\text { c- batido com cidreira }\end{array}$ \\
\hline $\begin{array}{l}\text { Losna - Artemisia } \\
\text { absinthium L. }\end{array}$ & Asteraceae & a- estômago, fígado & a- folhas & $\begin{array}{l}\text { a- infuso, maceração, } \\
\text { decocto }\end{array}$ \\
\hline $\begin{array}{l}\text { Macaé - Leonurus } \\
\text { sibiricus L. }\end{array}$ & Lamiaceae & a- reumatismo & a- folhas & a- infuso \\
\hline $\begin{array}{l}\text { Mandioca - Manihot } \\
\text { esculenta Crantz }\end{array}$ & Euphorbiaceae & a- reforço alimentar & a- farinha & a- farinha \\
\hline Manga - Mangifera indica L. & Anacardiaceae & $\begin{array}{l}\text { a- gripe, tosse } \\
\text { b- rins, infecção }\end{array}$ & $\begin{array}{l}\text { a- folhas } \\
\text { b- entrecasca }\end{array}$ & $\begin{array}{l}\text { a- melado } \\
\text { b- decocto }\end{array}$ \\
\hline $\begin{array}{l}\text { Maracujá - Passiflora } \\
\text { edulis Sims }\end{array}$ & Passifloraceae & a- pressão alta, diabete & a- folhas & a- decocto \\
\hline Marmelo - Pyrus cidonia L. & Rosaceae & a- rins & a- folhas & a- infuso \\
\hline $\begin{array}{l}\text { Melão de São Caetano - } \\
\text { Momordica charantia L. }\end{array}$ & Cucurbitaceae & a- malária & a- folhas & a- decocto \\
\hline $\begin{array}{l}\text { Mentrasto - Agerantum } \\
\text { conyzoides L. }\end{array}$ & Asteraceae & a- rins & a- folhas & a- infuso \\
\hline $\begin{array}{l}\text { Pariparoba - Pothomorphe } \\
\text { umbellata (L.) Miq. }\end{array}$ & Piperaceae & $\begin{array}{l}\text { a- hepatite, sangue, rins, } \\
\text { bronquite }\end{array}$ & a- folhas & a- decocto e infuso \\
\hline $\begin{array}{l}\text { Pata-de-vaca - Bauhinia } \\
\text { forficata Link }\end{array}$ & Leguminosae & $\begin{array}{l}\text { a- câncer, rins, diabete, } \\
\text { coração }\end{array}$ & a- folhas & a- decocto e infuso \\
\hline
\end{tabular}


Tabela 1. (Continuação)

\begin{tabular}{|c|c|c|c|c|}
\hline Nome vulgar - Científico & Família & Indicação $^{1}$ & Parte utilizada $^{1}$ & Modo de preparo ${ }^{1}$ \\
\hline $\begin{array}{l}\text { Pé-de-galinha - Eleusine } \\
\text { indica (L.) Gaertn }\end{array}$ & Gramineae & $\begin{array}{l}\text { a- pneumonia, bronquite, } \\
\text { asma } \\
\text { b- garganta }\end{array}$ & $\begin{array}{l}\text { a- folhas e raizes } \\
\text { b- folhas }\end{array}$ & $\begin{array}{l}\text { a- decocto } \\
\text { b- sumo }\end{array}$ \\
\hline Picão - Bidens pilosa L. & Asteraceae & a- malária, fígado & a- folhas e raízes & a- decocto \\
\hline $\begin{array}{l}\text { Pimenta - Capsicum } \\
\text { frutescens L. }\end{array}$ & Solanaceae & a- tumor b- pano branco ${ }^{6}$ & $\begin{array}{l}\text { a- folhas } \\
\text { b- folhas }\end{array}$ & $\begin{array}{l}\text { a- aplicar no local } \\
\text { b- limpar local com folhas }\end{array}$ \\
\hline Poejo - Mentha pulegium L. & Lamiaceae & $\begin{array}{l}\text { a- cólica, calmante } \\
\text { b- dor de barriga } \\
\text { c- gripe }\end{array}$ & $\begin{array}{l}\text { a- folhas, raízes } \\
\text { b- folhas, raízes } \\
\text { c- folhas, raízes }\end{array}$ & $\begin{array}{l}\text { a- infuso } \\
\text { b- decocto } \\
\text { c- melado }\end{array}$ \\
\hline $\begin{array}{l}\text { Quebra-pedra - } \\
\text { Phyllanthus niruri L. }\end{array}$ & Euphorbiaceae & a- infecção de rins & a- raízes & a- macerado $\mathrm{c} /$ vinho \\
\hline Romã - Punica granatum L. & Punicaceae & $\begin{array}{l}\text { a- disenteria } \\
\text { b-garganta }\end{array}$ & $\begin{array}{l}\text { a- casca do fruto } \\
\text { b- folhas, fruto }\end{array}$ & $\begin{array}{l}\text { a- decocto } \\
\text { b- decocto e infuso }\end{array}$ \\
\hline $\begin{array}{l}\text { Sabugueiro - Sambucus } \\
\text { australis Cham.\& Schltdl. }\end{array}$ & Caprifoliaceae & $\begin{array}{l}\text { a- sarampo } \\
\text { b- gripe, tosse }\end{array}$ & $\begin{array}{l}\text { a- folhas, flores } \\
\text { b- folhas }\end{array}$ & $\begin{array}{l}\text { a- decocto e infuso } \\
\text { b- infuso }\end{array}$ \\
\hline $\begin{array}{l}\text { Saião - Bryophyllum } \\
\text { pinnatum (Lam.) Oken }\end{array}$ & Crassulaceae & $\begin{array}{l}\text { a- anemia } \\
\text { b- inflamação, infecção } \\
\text { c- pressão alta, antibiótico }\end{array}$ & $\begin{array}{l}\text { a- folhas } \\
\text { b- folhas } \\
\text { c- folhas }\end{array}$ & $\begin{array}{l}\text { a- batido c/ mel } \\
\text { b- sumo } \\
\text { c- infuso }\end{array}$ \\
\hline $\begin{array}{l}\text { Tansagem - Plantago } \\
\text { major L. }\end{array}$ & Plantaginaceae & a- antibiótico, infecção & a- folhas & a- decocto e infuso \\
\hline $\begin{array}{l}\text { Terramicina - } \\
\text { Althernanthera dentata } \\
\text { (Moench) Stuchlik }\end{array}$ & Amaranthaceae & $\begin{array}{l}\text { a- infecção, anti- } \\
\text { inflamatório }\end{array}$ & a- folhas & a- decocto e infuso \\
\hline
\end{tabular}

tas medicinais revelaram que a maior parte foi adquirida por meio de livros, com média de 11,3(a) citações por entrevistado. As outras formas de obtenção de informações registradas foram menos relevantes: a aquisição de conhecimentos de pessoas conhecidas resultou em média de 7,6(b), a partir de parentes 6,0(b) e de pastorais 5,5(b). Embora as pastorais de saúde sejam atuantes nas comunidades, o interesse das pessoas pelas leituras referentes ao assunto é bastante significativo. Atualmente, há uma grande profusão de livros abordando o uso de plantas medicinais, a maioria relativamente simples em termos de elaboração, de fácil entendimento, e que podem ser adquiridos a baixo preço. A forma de aquisição dos conhecimentos etnobotânicos, nesse estudo, difere bastante do levantamento etnobotânico realizado por Ming \& Amaral Júnior (2005), na reserva extrativista "Chico Mendes", no Acre, em que todos os entrevistados afirmaram que o aprendizado foi repassado pelos pais.

No que se refere ao conhecimento das plantas medicinais em relação ao tempo de residência no local, a maior média aponta para a faixa entre 7 a 9 anos, sendo de 12,3 citações por entre- vistado. Porém, não ocorreu diferença significativa entre as faixas estudadas, não se observando uma tendência nos resultados que permitisse inferências entre o tempo de residência dos entrevistados no local e o seu conhecimento sobre as plantas medicinais utilizadas. Provavelmente, estas duas variáveis não interagiram devido ao fato de que a maioria das plantas utilizadas no município estudado não são nativas. Se este fosse o caso, seria de se esperar que os moradores com maior tempo de residência detivessem maior conhecimento sobre a utilização das plantas na medicina popular do local.

O gênero masculino apresentou significativamente mais informações sobre as plantas medicinais no município estudado. Enquanto os homens entrevistados apresentaram uma média de 16,4(a) citações por entrevistado, a média para as mulheres foi de 10,4(b). No trabalho realizado por Ming \& Amaral Júnior (2005), com seringueiros da reserva extrativista "Chico Mendes", foi constatado que os homens, devido ao maior contato com a floresta, tendem a ter maior conhecimento de plantas deste ecossistema.

Observou-se que a maioria dos entrevistados cultivava as plantas consumidas, indicando que estas eram ao menos parcialmente preservadas.

Como foi observado neste trabalho, o estudo do conhecimento etnobotânico de comunidades predominantemente voltadas para o setor primário em geral nos leva a duas vertentes: a primeira é a observação das estratégias que o homem utiliza para lidar com a natureza, tentando melhorar de alguma forma sua qualidade de vida e; a segunda, é a necessidade pungente de proteger o conhecimento ancestral, resgatando e registrando informações, de forma a perpetuá-las para as gerações futuras. Esta pesquisa fornecerá subsídio para estudos fitoquímicos e farmacológicos necessários para confirmar as propriedades terapêuticas da maioria das espécies estudadas e para verificar a toxicidade ou inocuidade das mesmas para a saúde humana.

\section{REFERÊNCIAS}

ALBUQUERQUE UP. 1998. Introdução à etnobotânica. Recife: Bagaço. 72p.

CAMARGO MTLA. 1998. Plantas medicinais e de rituais afro-brasileiros II: estudo etnofarmacobotânico. São Paulo: Ícone. 232p.

CASTRO HG; FERREIRA FA. 2000. Contribuição ao estudo das plantas medicinais: carqueja (Baccharis genistelloides). Viçosa: UFV. 102p. 
ELISABETSKY E. 2001. Etnofarmacologia como ferramenta na busca de substâncias ativas. In: SIMÕES CMO; SCHENKEL EP; GOSMAN G; MELLO JCP; MENTZ LA; PETROVICK PR (eds). Farmacognosia: da planta ao medicamento. $3^{\mathrm{a}}$ ed. Porto Alegre: UFSC. p. 91-104.

FERNANDES TM. 2004. Plantas Medicinais: memória da ciência no Brasil. Rio de Janeiro: Fiocruz. 260p.

GARLET TMB; IRGANG BE. 2001. Plantas medicinais utilizadas na medicina popular por mulheres trabalhadoras rurais de Cruz Alta, Rio Grande do Sul, Brasil. Revista Brasileira de Plantas Medicinais 4: 9-18.

IBGE - Instituto Brasileiro de Geografia e Estatistica. 2005, 17 de abril. Cidades @. Disponível em http://www.ibge.gov.br/ cidadesat/default.php

MARTINS ER; CASTRO DM; CASTELLANI DC; DIAS JE. 1995. Plantas medicinais. Viçosa: UFV. 220p.

MATOS FJA. 1997. O formulário fitoterápico do Professor Dias da Rocha. $2^{\mathrm{a}}$ ed. Fortaleza: EUFC. 124p.
MIGUEL MD; MIGUEL OG. 2000. Desenvolvimento de fitoterápicos. São Paulo: Robe Editorial. 115p.

MILLIKAN B. 1998. Zoneamento SócioEconômico-Ecológico no Estado de Rondônia: análise de um instrumento de ordenamento territorial na fronteira amazônica. Brasília: PNUD/PLANAFLORO. 105p.

MING LC; AMARAL JÚNIOR, A. 2005. Aspectos etnobotânicos de plantas medicinais na reserva extrativista "Chico Mendes". The New York Botanical Garden. Disponível em http://www.nybg.org/bsci/acre/www 1/ medicinal.html. Acessado em 25 de agosto de 2005.

PHILLIPS O; GENTRY AH. 1993. The useful plants of Tambopata, Peru. I. Statistical hypotheses tests with a new quantitative technique. Economic Botany 47: 15-32.

RADOMSKI MI; WISNIEWSKI C. 2004. Teores de elementos químicos hidrossolúveis em folhas de espinheira-santa (Maytenus ilicifolia) (Schrad.) Planch. Revista Brasileira de Plantas Medicinais 6: 65-68.
RODRIGUES VEG. 1998. Levantamento florístico e etnobotânico de plantas medicinais dos cerrados na região do Alto Rio Grande$M G$. Lavras: UFLA. 229p (Tese mestrado).

SANTOS MRA; INNECCO R. 2004. Adubação orgânica e altura de corte da erva-cidreira brasileira. Horticultura Brasileira 22: 182-185.

SILVA AJR; ANDRADE LHC. 2005. Etnobotânica nordestina: estudo comparativo da relação entre comunidades e vegetação na Zona do Litoral - Mata do Estado de Pernambuco, Brasil. Acta Botanica Brasilica 19: 45-60. 